\title{
¿Difieren las influencias personales, sociales, ambientales y psicológicas en la actividad física en base al tipo desplazamiento al centro escolar?*
}

\section{Does personal, social, environmental and psychological influences differ on Physical Activity depending on active school commuting type?}

\author{
Alberto Abarca Sos ${ }^{\mathrm{a}}$ \\ Universidad de Zaragoza, España \\ ORCID: http://orcid.org/0000-0002-9208-4824 \\ Laura Gallardo \\ Universidad de Zaragoza, España \\ ORCID: http://orcid.org/0000-0003-4795-5798 \\ Eduardo Generelo Lanaspa \\ Universidad de Zaragoza, España \\ ORCID: http://orcid.org/0000-0001-6972-3088 \\ José Antonio Julián Clemente \\ Universidad de Zaragoza, España \\ ORCID: http://orcid.org/0000-0002-7076-6621 \\ JaVier Zaragoza CASterad \\ Universidad de Zaragoza, España \\ ORCID: http://orcid.org/0000-0002-9761-1953
} a Autor de correspondencia. Correo electrónico:
aabarca@unizar.es

Para citar este artículo: Abarca-Sos, A., Gallardo, L. O., Generelo Lanaspa, E., Julián Clemente, J. A., \& Zaragoza Casterad, J. (2019). ¿Difieren las influencias personales, sociales, ambientales y psicológicas en la actividad física en base al tipo desplazamiento al centro escolar? Universitas Psychologica, 18(4), 1-11. h ttps://doi.org/10.11144/Javeriana.upsy18-4.dips

\section{RESUMEN}

El objetivo del estudio es conocer el papel de un desplazamiento activo o pasivo en un modelo con influencias económicas, como la ocupación del padre y de la madre, psicológicas, como la percepción individual de salud, y variables demográficas sobre la actividad física. 1618 adolescentes (734 chicas) de primero a cuarto curso de Educación Secundaria Obligatoria $\left(\mathrm{M}_{\text {edad }}=14.46 ; \mathrm{DT}=1.28\right)$, muestra representativa de Aragón $(3 \%)$, contestaron cuestionarios sobre las variables estudiadas. El modelo de ecuaciones estructurales mixto multigrupo para clases conocidas muestra que en aquellos adolescentes que se desplazan activamente al centro escolar, su salud percibida está muy relacionada con su actividad física, y con el nivel socioeconómico parental, mientras que la influencia de género y edad permanece constante entre subpoblaciones.

\section{Palabras clave}

desplazamiento activo; actividad física; salud percibida; ocupación profesional; adolescentes.

\section{ABSTRACT}

The study aim is to analyze the role of active vs. passive commuting in a model with socio economic influences, father and mother professional level, psychological influences, as perceived health, and demographic 
variables as gender and age related with physical activity. A representative sample of Aragón (3\%), 1618 adolescents (734 girls) between $1^{\mathrm{O}}$ and $4^{\mathrm{O}}$ years of Education Secondary Obligatory (Mean age $=14.46, \mathrm{SD}=1.28$ years) completed questionnaires to assess the studied variables. The mixture structural modeling multigroup with known classes shows that the perceived health of active commuting adolescents are strongly related with physical activity and socioeconomic status. Gender and age are related with physical activity in sedentary and active commuting groups.

Keywords

active commuting; physical activity; perceived health; occupational level; adolescents.

La actividad física (AF) en la adolescencia es fundamental para la adquisición de hábitos saludables y para un buen estado de salud (Sallis, Prochaska, \& Taylor, 2000), ya que al desarrollarse durante esta etapa, existe mayor probabilidad de continuar durante la edad adulta (Telama et al., 2005). La Organización Mundial de la Salud (OMS) ha señalado como prioridad que la promoción de la AF se realice través del desplazamiento activo, andando y en bicicleta (Organización Mundial de la Salud, 2018), por lo que plantear proyectos relacionados con esta variable es una oportunidad para aumentar los niveles de AF habitual en adolescentes (Rodríguez-López et al., 2013).

En España, aproximadamente un 60-70\% de los adolescentes se desplaza de forma activa al colegio (Villa-González et al., 2012; Zaragoza, Corral, Estrada, Abós, \& Aibar, 2019). De igual modo, en otros contextos también hay un amplio porcentaje que se desplaza andando o en bicicleta (Ikeda et al., 2018). Sin embargo, no existe acuerdo en señalar la relación entre el desplazamiento activo — sea este andando o en bicicleta- y los niveles de AF. La disonancia radica en la disparidad de resultados encontrados: asociaciones positivas (Chillón et al., 2010; Mendoza et al., 2011) y falta de relaciones (Davison, Werder, \& Lawson, 2008), mientras que otros estudios encontraron diferencias en función de la edad y el género (Rosenberg, Sallis, Conway, Cain, $\&$ McKenzie, 2006). Por tanto, en el presente estudio se pretende arrojar luz a las relaciones entre desplazamiento activo vs. desplazamiento pasivo y AF en adolescentes, basándonos en un constructo teórico que dé solidez a las relaciones de las variables.

En el marco del modelo social ecológico resulta fundamental entender los factores biológicos, ambientales y sociales que condicionan la práctica de AF y otras conductas asociadas (Spence \& Lee, 2003). Por tanto, la comprensión de la conducta de desplazamiento activo al colegio en los adolescentes se tiene que abordar estudiando variables en diferentes factores del modelo teórico, y añadiendo aspectos psicológicos que complementan la explicación de la conducta.

Desde una perspectiva biológica, investigaciones previas muestran que edad y género aparecen como las variables que tienen mayor repercusión en los niveles de $\mathrm{AF}$ de los adolescentes (Craggs, Coder, Van Sluijs, \& Griffin, 2011). Según señalan los mismos autores, está ampliamente comprobado que los chicos realizan más AF que las chicas, así como que la AF desciende con la edad en ambos géneros. En cambio, en los patrones de desplazamiento activo hasta ahora analizados, no se han encontrado diferencias por género, pues muestran patrones similares (Kallio, Turpeinen, Hakonen, \& Tammelin, 2016; Zaragoza et al., 2019. En relación con la edad, los resultados encontrados muestran una mayor contribución del desplazamiento activo a la práctica de $\mathrm{AF}$ en alumnado de secundaria (36\%) que en alumnado de educación primaria (23\%) (Martin, Boyle, Corlett, Kelly, \& Reilly, 2016). La falta de convergencia de los resultados entre desplazamiento activo, AF y variables biológicas resalta la carencia de investigaciones que permitan conocer los factores que determinan el desplazamiento activo y su relación con la AF.

En relación con una perspectiva social, el estatus socioeconómico de las familias puede ser determinante en el desplazamiento activo, y posee un gran peso en comportamientos relacionados con la salud, especialmente en adolescentes (Currie et al., 2008). En un estudio reciente en población española, se concluye que escolares en barrios con estatus socioeconómicos 
bajos realizan mayor número de desplazamientos activos que aquellos que presentan altos niveles (Molina-García \& Queralt, 2017). Estos datos indican que es importante el análisis de variables socioeconómicas en estudios sobre desplazamiento activo, donde la ocupación profesional de los padres y madres es una variable relevante en el contexto de la $\mathrm{AF}$, que permite así dilucidar la posible implicación del contexto socioeconómico más próximo en la AF y el tipo de desplazamiento en los adolescentes (Currie, Elton, Todd, \& Platt, 1997).

Desde una perspectiva ambiental, en el tipo de desplazamiento activo y en la AF, el entorno es una variable clave (Chillón et al., 2010; VillaGonzález, Ruiz, \& Chillón, 2016). La distinción habitual realizada se basa en distinguir ambiente urbano vs. rural (Guillamón, García Cantó, \& Pérez Soto, 2017). Los estudios que han relacionado la $\mathrm{AF}$ con el entorno han encontrado disparidad de resultados: mayor $\mathrm{AF}$ en entorno rural (Cruz, Lara, Zagalaz, \& Torres-Luque, 2014), mayor AF en entorno urbano (Guillamón et al., 2017), y por el contrario, otros estudios no han encontrado diferencias debidas al entorno (Bathrellou, Lazarou, Panagiotakos, \& Sidossis, 2007). Como se puede apreciar, no hay un consenso claro en la literatura con relación a dichas variables, por lo que analizarlas junto a la oportunidad de ser activo en el desplazamiento a la escuela puede ayudar a aportar más información en dicha relación. Esta situación hace ineludible tener en cuenta la influencia del entorno próximo de los adolescentes sobre la tipología de desplazamiento al centro escolar y la AF.

Desde una perspectiva psicológica, la salud percibida se corresponde con uno de los factores mayormente relacionados con la morbilidad y la mortalidad (Bailis, Segall, \& Chipperfield, 2003). Desde la estrecha relación entre la práctica de $\mathrm{AF}$ y la salud, el estudio Health Behaviour in SchoolAged Children (HBSC) (Iannotti et al., 2009) mostró relaciones positivas entre los niveles de AF y la salud percibida de los participantes, al evidenciar el componente consciente que supone la práctica de AF en la salud en aquellas personas que la realizan. En un contexto más concreto y cercano, el único estudio desarrollado en España (Chillón, Villén-Contreras, PulidoMarcos, \& Ruiz, 2016), y a la luz de las relaciones encontradas entre salud percibida y AF, los resultados no fueron significativos entre el desplazamiento activo como un tipo específico de AF y la salud percibida de los participantes. Las implicaciones derivadas de estos resultados sugieren que el desplazamiento activo comprende procesos diferentes a la AF todavía desconocidos.

Dada la relevancia, los resultados previos y las carencias encontradas en la investigación, el presente estudio pretende integrar, desde las diferentes perspectivas enunciadas, el modelo social ecológico, e incluir una variable psicológica. Los objetivos son los siguientes: a) conocer los patrones (modo y duración) de desplazamiento activo al colegio en una muestra representativa de adolescentes de Aragón, analizando las diferencias por género; b) analizar la relación de las variables estudiadas: $\mathrm{AF}$, tipo de desplazamiento activo, género, edad, salud percibida, ocupación laboral de madres y padres y entorno; c) analizar en función del tipo de desplazamiento al centro educativo, pasivo o activo, si hay diferencias entre los dos grupos con relación a la AF en las influencias biológicas (edad y género), ambientales (entorno), sociales (ocupación profesional de los padres y madres) y psicológicas (salud percibida). En función de los objetivos planteados y de la bibliografía previa, se enuncian las siguientes hipótesis: a) el modo de desplazamiento activo al centro escolar será superior al $50 \%$ en la población, donde en la mayoría de los caos, es menor a 30 minutos, y no encontraremos diferencias en función del género; b) se encontrarán relaciones significativas y positivas entre la AF y el desplazamiento activo, así como con otras de las variables analizadas; c) edad, género, salud percibida y ocupación profesional influirán de forma diferencial sobre la AF en función del tipo de desplazamiento. 


\section{Método}

\section{Participantes}

El estudio se realizó en población adolescente de la comunidad autónoma de Aragón. La muestra participante fue seleccionada mediante muestreo estratificado y aleatorio por género, provincia, entorno (rural o urbano), tipo de centro educativo (público o concertado) y curso escolar (de primero a cuarto de la ESO). Según el método de Dawson-Saunders y Trapp (1994), con un intervalo de confianza del 97 $\%$, la muestra de 1704 adolescentes obtenida se considera representativa (población final teórica por este método de 1049 participantes). Los cuestionarios incompletos fueron eliminados, y dieron lugar a una muestra final de 1618 adolescentes de 11 a 18 años (Medad $=14.46$, DT $=1.29 ; 54.6 \%$ chicos), de los cuales el $88 \%$ reside en un entorno urbano.

\section{Instrumentos}

\section{Actividad física}

La escala Assesment of Physical Activity Levels, APALQ (Ledent, Cloes, \& Pieron, 1997; versión Española de Zaragoza et al., 2012) mide AF incluyendo los cuatro escenarios en que se puede desarrollar (doméstico, transporte, escolar y ocio) mediante 5 ítems con 4 opciones de respuesta, que dan lugar a un índice de AF (IAF) continuo. Un ejemplo de ítem es "ihaces actividades físicodeportivas fuera del horario del colegio (en un club o de forma organizada)?".

\section{Desplazamiento activo}

Se midió el tipo de desplazamiento (coche, moto, autobús, bicicleta y andando) y la duración (menos de $5 \mathrm{~min}$, entre 5 y $15 \mathrm{~min}$, entre 15 y $30 \mathrm{~min}$, entre 30 y $60 \mathrm{~min}$ y más de $60 \mathrm{~min}$ ) del desplazamiento escolar a través de un ítem para cada cuestión, basados en los trabajos desarrollados en el estudio Avena
(Análisis y Valoración del Estado Nutricional de los adolescentes), desarrollado en diferentes ciudades españolas (Chillón et al., 2010). Se clasificaron los estudiantes en activos cuando se desplazaban habitualmente andando o en bicicleta, y como pasivos cuando lo hicieron en coche, moto o autobús.

Ocupación de los progenitores

Se registró mediante un ítem de respuesta abierta la categoría profesional de cada uno de los progenitores, en el que los sujetos tenían que describir a qué se dedicaban exactamente su padre y su madre. A continuación, se categorizó a los progenitores en cuatro niveles, según recomiendan Álvarez-Dardet, Alonso y Domingo (1995), también interpretable como un gradiente profesional.

Percepción de salud

La subescala percepción de salud del cuestionario Physical Self-Description Questionnaire PSDQ (Marsh, Richards, Johson, \& Roche, 1994; versión española de Marsh, Marco, \& Abcy, 2002) mide la percepción del sujeto sobre su estado general de salud y su capacidad de recuperación tras una enfermedad. La subescala consta de 8 ítems con respuesta tipo Likert de 1 a 6. Un ejemplo de ítem es "raramente estoy indispuesto o enfermo". Posteriormente, EsteveRodrigo (2004) amplió el rango de respuesta de la subescala, de 1 a 99 en cada ítem, pauta utilizada para este estudio. Los resultados mostraron una fiabilidad óptima de 0.77 y una ampliación de la validez discriminante del instrumento, por lo que ha sido la versión utilizada en el presente estudio.

\section{Procedimiento}

Tras la obtención de los permisos éticos de Universidad de Zaragoza y el Gobierno de Aragón, se recogieron los permisos de participación en los centros educativos. Además, progenitores y tutores otorgaron consentimiento 
de participación. Los cuestionarios fueron administrados por personal investigador en los centros educativos durante las clases. Los participantes fueron informados del carácter voluntario y confidencial de los datos.

\section{Análisis estadísticos}

Se utilizaron los programas SPSS 21.0 y Mplus 7.11 para los diferentes análisis efectuados. En relación con el primer objetivo, se realizaron estadísticos descriptivos y tablas de contingencia. En segundo lugar, las relaciones entre las variables estudiadas se analizaron mediante correlaciones bivariadas de Pearson. Finalmente, se implementó un modelo de ecuaciones estructurales mixto multi-grupo (mixture modeling with known clases) para los grupos de desplazamiento activo vs. pasivo en el centro escolar. La ventaja de esta metodología frente a un análisis multigrupo tradicional radica en que las categorías son tomadas como subgrupos de toda la muestra (Kim, Mun, \& Smith, 2014).

\section{Resultados}

La Tabla 1 ofrece los porcentajes de chicos y chicas que acuden al centro escolar en desplazamiento activo y pasivo, así como la duración de los mismos.

\section{Tabla 1}

Porcentaje de participantes para desplazamiento activo y pasivo en función del género y porcentaje de duración del desplazamiento para cada categoría

\begin{tabular}{llrr}
\hline Género & Desplazamiento & Duración (min) & Porcentaje \\
\hline \multirow{4}{*}{ Masculino } & Activo & Menos de 5 & 49.5 \\
& $68.8 \%$ & $5-30$ & 44.3 \\
\cline { 2 - 4 } & & $30-60$ & 6.2 \\
\cline { 2 - 4 } Femenino & Pasivo & Menos de 5 & 0 \\
& \multirow{2}{*}{ Fetivo } & $5-30$ & 99.4 \\
& \multirow{2}{*}{$67.4 \%$} & $30-60$ & 0.6 \\
\cline { 2 - 4 } & \multirow{2}{*}{ Pasivo } & Menos de 5 & 45.5 \\
& \multirow{2}{*}{$32.6 \%$} & $5-30$ & 49 \\
& & $30-60$ & 5.5 \\
\hline
\end{tabular}

El porcentaje de participantes que van al colegio en desplazamiento activo y pasivo es similar para ambos géneros, con valores superiores para el desplazamiento activo. Un dato de interés es que un alto porcentaje de los sujetos que realizan un desplazamiento activo tardan menos de 30 minutos en llegar al centro escolar.

Como muestra la Tabla 2, la AF es mayor para los chicos y desciende con la edad. Un aspecto destacado es que a mayores niveles de AF, los resultados muestran una mayor salud percibida, como muestra su relación positiva, también evidenciada entre la AF y la ocupación profesional únicamente del padre. El entorno (urbano vs. rural) no muestra relación con la $\mathrm{AF}$ o el tipo de desplazamiento de los adolescentes. Por último, los resultados muestran una falta de relación entre el tipo de desplazamiento al centro escolar y el resto de las variables.

\section{Tabla 2}

Matriz de correlaciones entre las variables objeto de estudio

\begin{tabular}{|c|c|c|c|c|c|c|c|c|}
\hline Variables & 1 & 2 & 3 & 4 & 5 & 6 & 7 & 8 \\
\hline 1. $\mathrm{AF}^{\mathrm{a}}$ & 1 & 0.01 & $-0.33^{* *}$ & $-0.18^{* *}$ & $0.16^{* *}$ & $0.07^{*}$ & $0.10^{* *}$ & -0.01 \\
\hline 2. Desplazamiento ${ }^{b}$ & & 1 & 0.01 & -0.05 & 0.01 & 0.02 & 0.03 & 0.04 \\
\hline 3. Género ${ }^{c}$ & & & 1 & -0.01 & $-0.07^{* *}$ & $-0.07 *$ & $-0.09 * *$ & 0.02 \\
\hline 4. Edad & & & & 1 & 0.05 & 0.01 & 0.05 & $-0.06^{*}$ \\
\hline 5. Salud percibida & & & & & 1 & $0.12^{* *}$ & $0.14^{* *}$ & $-0.08^{* *}$ \\
\hline 6. Ocupación padred & & & & & & 1 & $0.39^{* *}$ & $-0.1^{* *}$ \\
\hline 7. Ocupación madred & & & & & & & 1 & $-0.06^{*}$ \\
\hline 8. Entorno & & & & & & & & 1 \\
\hline
\end{tabular}

Nota. ${ }^{a}$ Puntuación máxima de 20 puntos. ${ }^{b}$

Tipo de desplazamiento esta categorizado como

0 pasivo y 1 activo. ${ }^{\mathrm{c}}$ Chicas categorizadas como 1 y chicos como 0 . d Puntuación de 1 (menor categoría profesional) y 4 (mayor categoría profesional). Las relaciones significativas corresponden a $* p<0.05 * * p<0.01$

Finalmente, en la Figura 1 se muestran los resultados del análisis de ecuaciones estructurales mixto multigrupo para clases conocidas: subpoblación de adolescentes que realizan un tipo de desplazamiento activo al colegio (izquierda) y aquellos que lo realizan de forma pasiva (derecha). El modelo fue sometido al test de invarianza parcial de medida, donde se obtuvo $\Delta \chi^{2}(d f=7)=89.01, p<0.001$, por lo que se pueden concluir diferentes relaciones en el modelo propuesto en función del tipo de desplazamiento activo vs. pasivo con la AF. 


\section{Figura 1}

Parámetros estandarizados del modelo y varianza explicada de AF

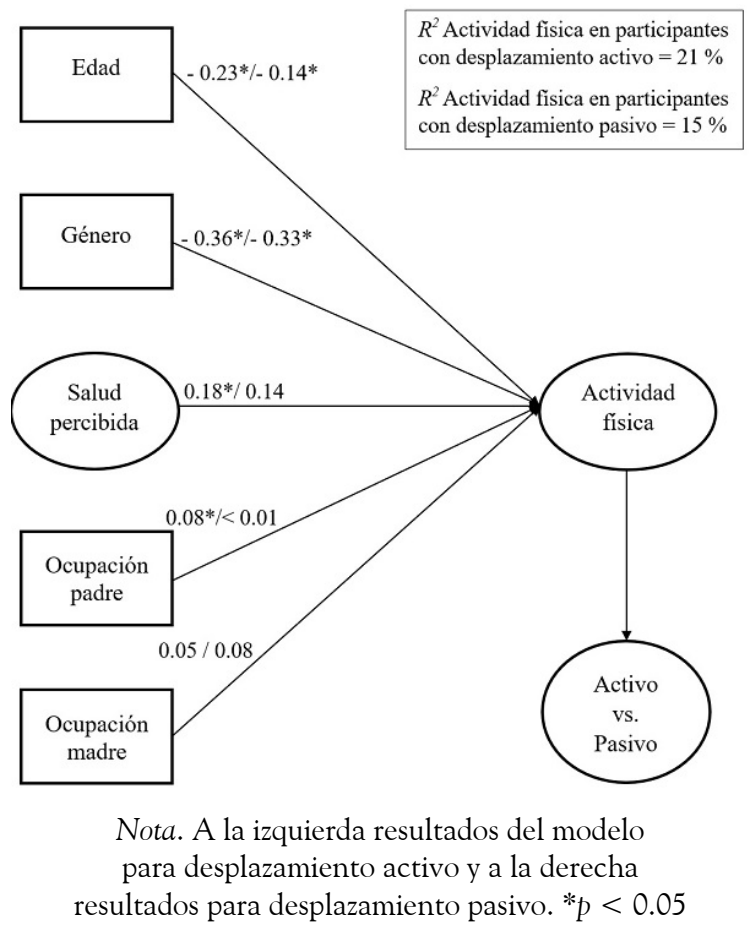

\section{Discusión}

Este estudio contribuye a mejorar el conocimiento sobre los patrones de desplazamiento activo al colegio, así como las influencias de diferentes variables del modelo social ecológico, añadiendo una psicológica en la $\mathrm{AF}$ en función de si los estudiantes se desplazan activa o pasivamente. En relación con la primera hipótesis, el estudio muestra que el $68 \%$ de los adolescentes acude al centro educativo de forma activa, y además no se han encontrado diferencias en función del género. Ambos resultados están en consonancia con otros estudios desarrollados en el contexto español (Zaragoza et al., 2019). Estos resultados reafirman que el tipo de desplazamiento al centro escolar de los adolescentes españoles es mayoritariamente activo y no está relacionado con el género, en contraposición a lo que ocurre con la AF (Faulkner, Builung, Flora, \& Fusco, 2009). Una posible explicación está relacionada con la distancia (Rodríguez-López et al., 2017). El tipo de desplazamiento vendrá influenciado por si los estudiantes viven lejos o cerca del centro educativo, no del género, ya que los progenitores favorecerán o no que sus hijos vayan al centro educativo de forma activa.

Respecto a la segunda hipótesis, ligada a la matriz de correlaciones de las variables, de forma contraria a lo esperado, la respectiva tabla muestra que la AF no está relacionada con el tipo de desplazamiento al centro escolar. Por ejemplo, Chillón et al. (2010) encontraron que aquellos adolescentes que se desplazan de manera activa al centro escolar registran un $6.8 \%$ más de AF que los que se desplazan de forma pasiva. Este porcentaje es relevante ya que muestra que el desplazamiento activo puede ser un tipo muy específico de AF que ayuda a compensar la posible carencia de realización de otras prácticas físicas (Mendoza et al., 2011). Sin embargo, parece que hay otras variables que sí están relacionadas con la $\mathrm{AF}$, como son las demográficas (género y edad), la salud percibida y las ocupaciones laborales del padre y de la madre. Estos resultados también se encuentran en el grupo de estudiantes que se desplaza de forma activa al realizar el modelo para ambos grupos, con excepción de la relación con la ocupación de la madre. Esta diferencia de las influencias en los diferentes tipos de análisis, la relación significativa en las correlaciones y la no relación en el modelo pueden estar vinculadas a que en las ecuaciones estructurales se incorporan las variables de forma conjunta, y se evalúan las complejas interrelaciones entre las mismas, aparte de integrar los errores de medida al mismo tiempo (Cupani, 2012), cuestión que en las correlaciones no se lleva a cabo. Por lo tanto, al haber controversia en los resultados, en las investigaciones de influencias sociales habrá que medir por separado a los padres y a las madres para seguir aportando información al respecto.

Siguiendo con la tercera hipótesis, en primer lugar, se debe señalar que el modelo testado ha seguido las relaciones hipotetizadas en el constructo teórico del modelo Social Ecológico (Spence \& Lee, 2003). En segundo lugar, se ha observado que, contando con el mismo 
conjunto de variables predictoras, la AF viene explicada en mayor medida en aquellos que se desplazan de forma activa. En conjunto, el desplazamiento activo parece ser un tipo específico de AF, cuya determinación e influencia vienen configuradas por procesos diferentes (Villa-Gónzalez et al., 2016). En el análisis de las posibles relaciones diferenciales desde una perspectiva social ecológica y psicológica, los resultados del modelo han mostrado que la tipología de desplazamiento - activa o pasivaproduce cambios en las relaciones establecidas, especialmente en variables psicológicas y sociales al determinar la AF en adolescentes. Concretamente, se ha encontrado que la salud percibida influencia la $\mathrm{AF}$ en aquellos adolescentes que se desplazan de forma activa al centro escolar. Este comportamiento diferencial podría explicar el hecho de que la salud percibida esté relacionada con la AF (Iannotti et al., 2009; Pastor, Balaguer, Pons, \& García-Merita, 2003), aunque no lo está de forma directa con el tipo de desplazamiento (Chillón et al., 2016). Las implicaciones de la relación encontrada entre salud percibida y AF en el grupo que se desplaza de forma activa apunta a que aquellas personas más activas tienen una percepción más positiva de su salud, lo que soporta la implementación de proyectos de intervención para fomentar el desplazamiento activo al colegio como mejora de la salud de los escolares.

En cuanto al nivel socioeconómico como influencia de la AF, los resultados del modelo apoyan que existe una mayor práctica de AF en las familias con mejores niveles socioeconómicos (Simpson, Janssen, Craig, \& Pickett, 2005). En nuestro estudio, la influencia existe por parte del padre en aquellos adolescentes que se desplazan de forma activa. El hecho de que se haya encontrado que existe una influencia socioeconómica paterna sobre la AF, solo sobre los desplazados activamente, permite arrojar luz ante la discrepancia de resultados encontrados previamente. Además, estudios previos que han tratado las influencias familiares, también de forma diferencial, han encontrado una influencia paterna del apoyo social con relación a la AF, y no así con relación a la madre (Abarca-Sos, Bois,
Zaragoza, Generelo, \& Julián, 2013). También cabe la posibilidad de que el comportamiento diferencial que ha mostrado la influencia socioeconómica entre los progenitores sobre la AF se pueda deber a las innumerables formas utilizadas de medir el nivel socioeconómico y el contexto del estudio (Currie et al., 2008). Estos hallazgos suponen que las influencias de los progenitores se tienen que medir de forma separada, cuando la mayoría de estudios lo hace forma conjunta, padre y madre, ya que los resultados entre ambos agentes pueden variar.

En cuanto a las variables biológicas, género y edad han cumplido los patrones habituales en las relaciones de investigaciones previas: la $\mathrm{AF}$ es mayor en los chicos que en las chicas, y disminuye a medida que aumenta la edad (Craggs et al., 2011), con valores similares en adolescentes que se desplazan activa y pasivamente. Es decir, independientemente de la forma de desplazamiento activo, dentro de cada grupo, los valores en la $\mathrm{AF}$ son superiores en los chicos, lo que implica que los programas de intervención para aumentar la AF tendrán que focalizar su atención en implementar estrategias en el género femenino (Murillo et al., 2013).

Existen una serie de limitaciones que han de ser consideradas en relación con el estudio presentado. La primera sería la utilización de un cuestionario para medir la AF, ya que conocer sus niveles a través de métodos precisos de evaluación, como por ejemplo acelerometría, puede ayudar a objetivizar los datos. Futuras investigaciones podrían utilizar medidas objetivas y subjetivas para dilucidar la relación entre AF y el desplazamiento activo en otras variables. Encontramos otra limitación por la naturaleza transversal del estudio, ya que no se pueden establecer relaciones de causalidad entre los resultados encontrados. Futuras investigaciones estarían encaminadas a conseguir datos longitudinales que permitan conocer la estabilidad de las relaciones y el establecimiento de causalidad. Finalmente, el modelo estructural analizado parte del modelo social ecológico, que integra una variable psicológica, sin embargo, otras variables podrían ser contempladas: distancia al centro educativo, 
barreras percibidas por los padres y madres o peligrosidad del barrio, las cuales han sido determinantes en otros estudios. Además, futuras investigaciones podrían explorar las posibles variables mediadoras entre el desplazamiento activo vs. pasivo y la AF, así como los procesos subyacentes compartidos.

\section{Conclusiones}

Aproximadamente un $60 \%$ de los adolescentes aragoneses se desplaza de forma activa, ya sea caminando o en bicicleta, al centro escolar de forma habitual, independientemente de su género. Al desplazamiento activo al centro escolar, entendido como un tipo específico de AF, le subyacen determinantes y consecuencias diferenciales. Así, una perspectiva social ecológica, donde se recogen variables sociodemográficas, sociales y ambientales añadiendo psicológicas - influye de forma diferencial sobre la AF de los adolescentes en función del desplazamiento activo vs. pasivo de los mismos al centro escolar. Conocer los procesos diferenciales y comunes del desplazamiento activo y la $\mathrm{AF}$ nos permitirá redundar en mayores niveles de la misma, y por ende en la salud en los adolescentes.

\section{Referencias}

Abarca-Sos, A., Bois, J. E., Zaragoza, J., Generelo, E., \& Julian, J. A. (2013). Ecological correlates of physical activity in Routh: Importance of parents, friends, physical education teachers and geographical localization. International Journal of Sport Psychology, 44(3), 215-233.

Álvarez-Dardet, C., Alonso, J., \& Domingo, A. (1995). La medición de la Clase social en Ciencias de la Salud. Barcelona: SG Editores-SEE.

Bailis, D., Segall, A., \& Chipperfield, J. G. (2003). Two views of self-rated general health status. Social Science $\mathbb{E}$ Medicine, 56(2), 203-217. https://doi.org/10.1016/SO 277-9536(02)00020-5
Bathrellou, E., Lazarou, C., Panagiotakos, D. B., \& Sidossis, L. S. (2007). Physical activity patterns and sedentary behaviors of children from urban and rural areas of cyprus. Central European Journal of Public Health, 15(2), 66-70.

Chillón, P., Ortega, F. B., Ruiz, J. R., Veidebaum, T., Oja, L., Mäestu, J., \& Sjöström, M. (2010). Active commuting to school in children and adolescents: An opportunity to increase physical activity and fitness. Scandinavian Journal of Public Health, 38(8), 873-879. https://doi.org/10.1177/14034948 10384427

Chillón, P., Villén-Contreras, M., Pulido-Marcos, M., \& Ruiz, J. R. (2016). Desplazamiento activo al colegio, salud positiva y estrés en niños españoles. SportTK: Revista Euroamericana de Ciencias del Deporte, 6(1), 117-124. https://doi.org/10.6018/280521

Craggs, C., Corder, K., Van Sluijs, E. M., \& Griffin, S. J. (2011). Determinants of change in physical activity in children and adolescents: A systematic review. American Journal of Preventive Medicine, 40(6), 645-658. https://doi.org/10.1016/j.a mepre.2011.02.025

Cruz, A., Lara, A., Zagalaz, M. L., \& TorresLuque, G. (2014). Analysis and Evaluation of the Fitness of Primary School Students in Rural and Urban Areas. Apunts. Educación Física y Deportes, 116(2), 44-51.

Cupani, M. (2012). Análisis de Ecuaciones Estructurales: conceptos, etapas de desarrollo y un ejemplo de aplicación. Revista Tesis, 1(1), 186-199.

Currie, C. E., Elton, R. A., Todd, J., \& Platt, S. (1997). Indicators of socioeconomic status for adolescents: th WHO Health Behaviour in School-aged Children Survey. Health Education Research, 12 (3), 385-397. https:// doi.org/10.1093/her/12.3.385

Currie, C., Molcho, M., Boyce, W., Holstein, B., Torsheim, T., \& Richter, M. (2008). Researching health inequalities in adolescents: The development of the Health Behaviour in School-Aged Children (HBSC) Family Affluence Scale. Social 
Science Ė Medicine, 66(6), 1429-1436. https ://doi.org/10.1016/j.socscimed.2007.11.024

Davison, K. K., Werder, J. L., \& Lawson, C. T. (2008). Children's active commuting to school: current knowledge and future directions. Preventing Chronic Disease, 5(3), 701-704.

Dawson-Saunders, B., \& Trapp, R. G. (1994). Basic and Clinical Biostatistics ( ${ }^{\mathrm{a}}$ ed.). Norwalk, CT: Appleton \& Lange.

Esteve-Rodrigo, J. V. (2004). Estilos parentales, clima familiar y autoestima física en adolescentes (Tesis Doctoral). Universidad de Valencia, España.

Faulkner, G. E. J., Buliung, R. N., Flora, P. K., \& Fusco, C. (2009). Active school transport, physical activity levels and body weight of children and youth: A systematic review. Preventive Medicine, 48(1), 3-8. https://doi. org/10.1016/j.ypmed.2008.10.017

Guillamón, A. R., García Cantó, E. G., \& Pérez Soto, J. P. (2017). Diferencias en la condición física en escolares de entornos rurales y urbanos de Murcia (España). Revista de Estudios y Experiencias en Educación, 16(30), 115-128. https://doi. org/10.21703/rexe.2017301151286

Iannotti, R. J., Janssen, I., Haug, E., Kololo, H., Annaheim, A., \& HBSC Physical Activity Focus Group. (2009). Interrelationships of adolescent physical activity, screenbased sedentary behavior, and social and psychological health. International Journal of Public Health, 54(2), 191-198. https://doi.o $\mathrm{rg} / 10.1007 / \mathrm{s} 00038-009-5410-z$

Ikeda, E., Stewart, T., Garrett, N., Egli, V., Mandic, S., Hosking, J., ...Witten, K. (2018). Built environment associates of active school travel in New Zealand children and youth: A systematic metaanalysis using individual participant data. Journal of Transport EF Health, 9, 117-131. h ttps://doi.org/10.1016/j.jth.2018.04.007

Kallio, J., Turpeinen, S., Hakonen, H., \& Tammelin, T. (2016). Active commuting to school in Finland, the potential for physical activity increase in different seasons. International Journal of Circumpolar Health,
75(1). https://doi.org/10.3402/ijch.v75.333 19

Kim, S. Y., Mun, E. Y., \& Smith, S. (2014). Using mixture models with known class membership to address incomplete covariance structures in multiple-group growth models. British Journal of Mathematical and Statistical Psychology, 67(1), 94-116. https://doi.org/1 0.1111/bmsp.12008

Ledent, M., Cloes, M., \& Pierón, M. (1997). Les jeunes, leur activite physique et leurs perceptions de la sante, de la forme, des capacites athletiques et de l'apparence. Sport, 40(3), 90-95.

Marsh, H. W., Marco, I. T., \& Abcy, F. H. (2002). Cross-cultural validity of the physical selfdescription questionnaire: comparison of factor structures in Australia, Spain, and Turkey. Research Quarterly in Exercise and Sport, 73(3), 257-270. https://doi.org/10.10 80/02701367.2002.10609019

Marsh, H. W., Richards, E., Johson, S., \& Roche, L. (1994). Physical Self-Description Questionnaire: Psychometric properties and a multitrait-multimethod analysis of relations to existing instruments. Journal of Sport Exercise Psychology, 16(3), 270-305.

Martin, A., Boyle, J., Corlett, F., Kelly, P., \& Reilly, J. J. (2016). Contribution of walking to school to individual and population moderate-vigorous intensity physical activity: systematic review and meta-analysis. Pediatric Exercise Science, 28(3), 353-363. https://doi.org/10.1123/pes $.2015-0207$

Mendoza, J. A., Watson, K., Nguyen, N., Cerin, E., Baranowski, T., \& Nicklas, T. A. (2011). Active commuting to school and association with physical activity and adiposity among US youth. Journal of Physical Activity and Health, 8(4), 488-495.

Molina-García, J., \& Queralt, A. (2017). Neighborhood built environment and socioeconomic status in relation to active commuting to school in children. Journal of Physical Activity and Health, 14(10), 
761-765. https://doi.org/10.1123/jpah.2017 $-0033$

Murillo, B., García-Bengoechea, E., Generelo, E., Bush, P. L., Zaragoza Casterad, J., Julián, J. A., \& García González, L. (2013). Promising school-based strategies and intervention guidelines to increase physical activity of adolescents. Health Education Research, 28(3), 523-538. https:// doi.org/10.1093/her/cyt040

Organización Mundial de la Salud. (2018). Global Action Plan on Physical Activity 2018-2030: More Active People for a Healthier World. Ginebra: Organización Mundial de la Salud. Recuperado de http://apps.who.int/iris/bitstream/handl e/10665/272722/9789241514187-eng.pdf

Pastor, Y., Balaguer, I., Pons, D., \& Garcia-Merita, M. (2003). Testing direct and indirect effects of sports participation on perceived health in Spanish adolescents between 15 and 18 years of age. Journal of Adolescence, 26(6), 717-730. https://doi.org/10.1016/j.a dolescence.2003.07.001

Rodríguez-López, C., Salas-Fariña, Z. M., VillaGonzález, E., Borges-Cosic, M., HerradorColmenero, M., Medina-Casaubón, J., ... Chillón, P. (2017). The threshold distance associated with walking from home to school. Health Education Behaviour, 44(6), 857-866. https://doi.org/10.1177/10901981 16688429

Rodríguez-López, C., Villa-González, E., PérezLópez, I. J., Delgado-Fernández, M., Ruiz, J. R., \& Chillón, P. (2013). Los factores familiares influyen en el desplazamiento activo al colegio de los niños españoles.Nutrición Hospitalaria, 28(3), 756-763. https://doi.org/10.3305/nh .2013.28.3.6399

Rosenberg, D. E., Sallis, J. F., Conway, T. L., Cain, K. L., \& McKenzie, T. L. (2006). Active transportation to school over 2 years in relation to weight status and physical activity. Obesity, 14(10), 1771-1776. https:/ /doi.org/10.1038/oby.2006.204

Sallis, J. F., Prochaska, J. J., \& Taylor, W. C. (2000). A review of correlates of physical activity of children and adolescents. Medicine and Science in Sports and Exercise, 32(5), 963-975. https://doi.org/0195-9131/ 00/3205-0963/0

Simpson, K., Janssen, I., Craig, W. M., \& Pickett, W. (2005). Multilevel analysis of associations between socioeconomic status and injury among Canadian adolescents. Journal of Epidemiology $\&$ Community Health, 59(12), 1072-1077. https://doi.org/ 10.1136/jech.2005.036723

Spence, J. C., \& Lee, R. E. (2003). Toward a comprehensive model of physical activity. Psychology of sport and exercise, 4(1), 7-24. https://doi.org/10.1016/S1469-0292( 02) $00014-6$

Telama, R., Yang, X., Viikari, J., Valimaki, I., Wanne, O., \& Raitakari, O. (2005). Physical activity from childhood to adulthood: A 21-year tracking study. American Journal of Preventive Medicine, 28(3), 267-273. https://doi.org/10.1016/j.a mepre.2004.12.003

Villa-González, E., Rodríguez-López, C., Huertas, F. J., Tercedor, P., Ruiz, J. R., \& Chillón. P. (2012). Factores personales y ambientales asociados con el desplazamiento activo al colegio de los escolares españoles. Revista de Psicología del Deporte, 21 (2), 343-349.

Villa-González, E., Ruiz, J., \& Chillón, P. (2016). Recomendaciones para implementar intervenciones de calidad de promoción del desplazamiento activo al colegio. Retos, 30, 159-161.

Zaragoza, J., Corral, A., Estrada, S., Abós, Á., \& Aibar, A. (2019). Active or passive commuter? Discrepancies in cut-off criteria among adolescents. International journal of environmental research and public health, 16(20), 3796. https://doi.org/10.3390/ijerp h16203796

Zaragoza, J., Generelo, E., Aznar, S., AbarcaSos, A., Julián, J. A., \& Mota, J. (2012). Validation of a short physical activity recall questionnaire completed by spanish adolescents. European Journal of Sport Sciences, 12(3), 283-291. https://doi.org/10. 1080/17461391.2011.566357 


\section{Notas}

* Artículo de investigación. 